\title{
Estructura interna de la Escala de Conciliación Vida-Trabajo para mujeres asalariadas del noreste de México
}

\section{Internal Structure of the Conciliation Scale of Family and Work Life, for women who are in paid works in the north-east of Mexico}

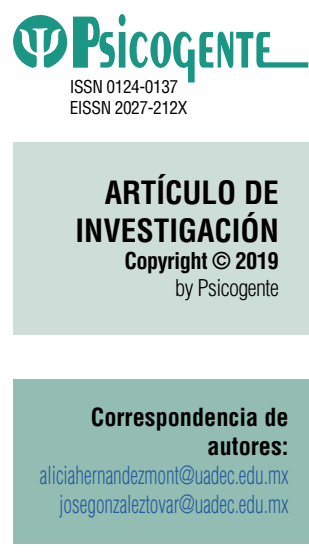

Recibido: 08-02-18 Aceptado: $10-10-18$ Publicado: 01-07-19

\author{
Alicia Hernández-Montaño iD \\ Universidad Autónoma de Coahuila, Saltillo, México \\ José González-Tovar ID \\ Universidad Autónoma de Coahuila, Saltillo, México
}

Resumen

Objetivo: Realizar la validación inicial de la estructura de la Escala de Conciliación Vida-Trabajo en mujeres insertas en el mercado laboral del noreste de México.

Método: Se utilizó un diseño cuantitativo de corte explicativo. El muestreo fue intencional, 554 mujeres mayores de 18 años y que desempeñan actividades laborales remuneradas. El instrumento contó con 35 reactivos organizados en ocho dimensiones: desarrollo personal, vida familiar, vida social, cuidado personal, alimentación, actividad física, satisfacción e interferencia.

Resultados: Se obtuvo la consistencia interna y el análisis factorial exploratorio (AFE) y confirmatorio (AFC). Se logró una reducción de ocho a seis dimensiones y de 35 a 25 reactivos. Una vez obtenidos estos valores, la escala tiene una adecuada estructura interna $(\mathrm{GFI}=0,867, \operatorname{RMSEA}=0,063)$ es válida y confiable $(\alpha=0,874)$ para evaluar el nivel de conciliación vida-familia de mujeres que se desempeñan en diferentes sectores laborales.

Conclusiones: La gratificación, interferencia, pareja y crianza, cuidado de la salud, responsabilidades en el hogar y tiempo libre son dimensiones de la ECVT que permiten identificar la estructura de la conciliación desde la perspectiva de las mujeres trabajadoras.

Palabras clave: determinantes sociales de salud, mujeres trabajadoras, género.

Abstract

Objective: Validation of the Conciliation Scale of Family and Work Life, for women who are in the north-east of Mexico labor market.

Method: In this research, a quantitative and explanatory design was developed. 554 women over 18 years who are in paid works were intentionally sampled. An instrument of 35 reagents organized in eight dimensions such as: personal development, family life, social life, personal care, food, physical activity, satisfaction and interference was used.

Results: Internal consistency, exploratory factorial analysis (AFE) and confirmatory analysis (AFC) were performed. There was a reduction from eight to six dimensions and from 35 to 25 reagents. As soon as these values were obtained, this scale has an appropriate structure as follows: $(G F I=0,867, R M S E A=0,063)$, becoming valid and reliable $(\alpha=0,874)$ to assess the level of reconciliation between women and their Family and Work Life.

Conclusions: Gratification, interference, couple and upbringing, health care, responsibilities in the home and free time are dimensions of the ECVT that allow to identify the structure of the conciliation from the perspective of working women.

Key words: social determinants of health, working women, gender.

Cómo citar este artículo (APA):

Hernández-Montaño, A. \& González-Tovar, J. (2019). Estructura interna de la Escala de Conciliación Vida-Trabajo para mujeres asalariadas del noreste de

México. Psicogente 22(42), 1-18. https://doi.org/10.17081/psico.22.42.3472 


\section{INTRODUCCIÓN}

A lo largo de su vida laboral, las mujeres siguen experimentando dificultades para crecer profesionalmente, o incluso a acceder a empleos dignos. La desigualdad entre mujeres y hombres persiste en los mercados laborales mundiales en lo que respecta a las oportunidades y a la calidad de los empleos, que muchas veces son mal remunerados y sin protección social (Díaz, Dema \& Finkel, 2015; Prieto \& Pérez, 2013; Worth, 2016). El trayecto laboral de las mujeres está caracterizado también por problemas de acoso sexual, alto esfuerzo y/o baja recompensa, exponiéndose a más riesgos psicosociales y sufriendo mayor discriminación (Campos, Ronda, Artazcoz \& Benavides, 2012).

Entre 1995 y 2015, la tasa mundial de participación de las mujeres en la fuerza de trabajo cayó del 52,4 \% al 49,6\%. A nivel mundial, la probabilidad de que las mujeres participen en el sector laboral sigue siendo de casi 27 puntos porcentuales menores que el hombre (Organización Internacional del Trabajo [OIT], 2016).

Para América Latina el promedio de participación de las mujeres en la fuerza laboral es del $30 \%$, dato por debajo del $43,8 \%$ que representa a las mujeres trabajadoras en México (Instituto Nacional de Estadística y Geografía [INEGI], 2016). Los salarios de las mexicanas son $50,5 \%$ menores en los niveles de supervisión industrial, 16,1 \% en los de profesionales y 31,3 \% en el sector público. Solamente en los trabajos domésticos los ingresos de las mujeres superan los de los varones en 7,4 \% (INEGI-INMUJERES, 2007). Estos datos muestran que su acceso a la vida productiva no ha sido equitativo, pues sus salarios y condiciones laborales difieren por mucho de los varones.

El continuo crecimiento de la incorporación de las mujeres al mercado laboral ha representado nuevos estilos de vida que han afectado directamente la organización familiar. Para las mujeres ha implicado la modificación de su tiempo diario al tener que compaginar su presencia fuera y dentro del hogar, situación que no se ve reflejada en la vida de los hombres (Hernández, 2016; Rocha, 2013). Hay que destacar que no ha sido una tarea fácil, pues estos cambios no modificaron, en lo substancial, la división sexual del trabajo, es decir, las mujeres cumplen con sus jornadas de trabajo y continúan en casa con las tareas de cuidado y atención de la familia (Hernández et al., 2017).

Algunos ejemplos son los estudios en población con mujeres que desempeñan actividades remuneradas (Alarcón, 2012; Feldman, Vivas, Lugli, Zaragoza \& 
Gómez, 2008) que destacan la prevalencia de una división sexual de trabajo que asigna al hombre la responsabilidad de la provisión de ingresos familiares y a las mujeres las obligaciones de reproducción del mundo doméstico, incluyendo el cuidado y la crianza de hijos e hijas.

Diversos autores (Hernández, González, López \& Saucedo, 2015; López et al., 2011; Tena, 2013) ponen de manifiesto la escasa participación de los varones en las tareas domésticas, la mayoría no las asume en forma sistemática, con frecuencia tiene la forma de ayuda o colaboración -fines de semana, vacaciones, en caso de enfermedad- y con mayor regularidad cuando las esposas se incorporan a la vida productiva.

Además de que cuando estos participan lo hacen de las tareas menos penosas, que no requieren una rutina y por lo tanto, tampoco de una presencia constante; por ejemplo, tan solo la preparación y servicio de alimentos para los miembros del hogar les lleva a las mujeres un promedio de 15 horas a la semana (contra 4 horas de los hombres), actividad vital que no puede dejarse para después, como lo pudiera ser la reparación de bienes o pago de servicios, que es donde se registra una mayor participación de los varones (INEGI, 2011; Rocha, 2013). El ámbito del cuidado y crianza de hijos/as ha registrado diversos cambios, un mayor involucramiento de los varones en estas funciones cuando las madres salen a trabajar fuera de casa (Guevara, 2010).

Lo anterior sugiere la necesidad de conciliar los escenarios públicos y las tareas domésticas y el cuidado de hijos/as en mujeres trabajadoras. Para este estudio retomaremos la definición de Tena (2013), quien señala que la reconciliación es la necesidad de armonizar el tiempo dedicado al trabajo y la vida familiar, como una estrategia para garantizar la participación de las mujeres en los empleos, sin que esto les represente una doble carga de trabajo que termine afectando su salud física y mental. McMillan, Morris y Atchley (2011) han ampliado el concepto de trabajo y familia para incluir la vida personal, entendida como aquellas actividades que involucran el cuidado o recreación individual de las mujeres, relacionadas con su salud y la vida social.

A su vez, las organizaciones han adoptado iniciativas de conciliación trabajo-familia, también llamadas prácticas flexibles, como: reducción de jornada, permisos, horario flexible, etc. con la finalidad de generar condiciones para que los trabajadores aumenten su satisfacción laboral y reduzcan los conflictos familiares (Pérez, Vela, Abella \& Martínez, 2015). Sin embargo, muchas veces 
estas iniciativas están dirigidas en mayor medida al sector femenino con la intención de apoyarlas para no descuidar sus responsabilidades familiares, lo que lo hace ver como un problema principalmente de este sector, planteamiento que obstaculiza la transición hacia prácticas menos estereotipadas y la concreción de una distribución más equitativa de roles en la familia y las organizaciones. Las investigadoras Jiménez y Gómez (2015) han planteado que el tema de la conciliación va más allá de un problema privado que contempla el bienestar subjetivo, la satisfacción laboral y otros indicadores de la calidad de vida; su mayor aporte es en términos de una equidad social y de género que promueva la igualdad de oportunidades entre hombres y mujeres en las diferentes esferas de vida.

Un aspecto importante de la conciliación laboral-personal es el número de horas que una persona trabaja. La Organización para la Cooperación y el Desarrollo Económico (OCDE, 2015) sugiere que un horario de trabajo largo puede resultar perjudicial para la salud, poner en peligro la seguridad y aumentar el estrés. En México el $35 \%$ de los hombres trabajan con un horario muy largo, en comparación con el 18 \% del caso de las mujeres (Organización para la Cooperación y el Desarrollo Económico [OCDE], 2015). Sin embargo, estas últimas invierten más tiempo en las tareas del hogar y crianza de los hijos, lo que hace que la mayoría de ellas finalmente cumpla con jornadas largas de trabajo.

Este escenario nos ayuda a comprender el impacto negativo en la salud de las mujeres, quienes se enfrentan a jornadas interminables para cumplir con las cargas derivadas de su rol dependiente y de cuidadora, lo que sugiere la necesidad de estudiar este fenómeno desde una perspectiva de género.

\subsection{Trabajo reproductivo vs trabajo productivo}

Cuando las mujeres se incorporan al sector laboral lo hacen con una identidad de género socializada en valores femeninos vinculados con el cuidado y el ser para los demás (Alcaniz, 2015). Una internalización de su "deber ser" basado en estereotipos de género asociados con mayor regularidad a características como la sensibilidad, el amor, la complacencia, la entrega, la fidelidad y el romanticismo, estereotipos idóneos para responsabilizar y justificar sus labores de maternidad y convivencia en el ámbito familiar (Izquierdo, 2013; Tobío, 2012).

Esto nos ayuda a comprender que la supuesta especialización de las mujeres en el campo reproductivo y la del varón en el campo productivo, se debe a 
una serie de creencias sociales y culturales que nos hacen suponer que cada uno de estos actores posee ciertas cualidades o habilidades "innatas" con base en el sexo biológico al que pertenecen.

De ahí que la división más básica es la que separa el trabajo reproductivo del trabajo productivo. El primero de ellos es donde las mujeres tienen un papel protagónico, cumplen con tareas relacionadas al ámbito doméstico y al cuidado de la familia, principalmente de los hijos, adultos mayores y enfermos; carece de un reconocimiento social, son actividades cíclicas y cotidianas, no tiene horarios, vacaciones, no es remunerado económicamente y se realizan a lo largo de todo el ciclo de la vida. Sagastizabal y Legarreta (2016) observan que, en los últimos veinte años, el incremento de las mujeres en la vida productiva disminuyó su intervención en el trabajo doméstico, probablemente a la disminución en la disponibilidad de su tiempo, pero no pasó así con el cuidado, por lo que afirman que el espacio de cuidado se reafirma como espacio de la feminidad. El segundo, el trabajo no doméstico o productivo, es un espacio tradicionalmente masculino, son acciones vinculadas con la producción y la política, cuenta con un reconocimiento social, producen bienes o servicios y tienen un valor de cambio. Se da dentro de un horario específico, generalmente en la semana, con descansos el fin de semana y se efectúa hasta alcanzar la jubilación (Benería, 2006). A pesar de que existe una mayor predisposición a participar en el ámbito doméstico-familiar los datos solo muestran un incremento de 15 minutos más para el trabajo doméstico, sin contar los cuidados (Sagastizabal \& Legarreta, 2016).

Son estructuras de orden de género que han sufrido cambios poco significativos al momento en que las mujeres se han incorporado a la escena productiva, mismas que limitan las intenciones y esfuerzos de los individuos para modificar significativamente las relaciones de género dentro del hogar (Bamberry, 2016).

De esta manera, las construcciones de género no solo conllevan a la repartición de quehaceres diferentes para hombres y mujeres, sino que instituyen y promueven la desigualdad e injusticia, con mayor desventaja y repercusión en la vida de las mujeres.

Un ejemplo es la doble carga de trabajo que muchas mujeres alrededor del mundo tienen que sortear. En los países tanto de altos ingresos como de bajos ingresos, las mujeres siguen trabajando menos horas en un empleo remunerado, mientras que asumen la gran mayoría de las labores de cuidado 
y las tareas domésticas no remuneradas. En promedio, en las naciones en las que existen datos pertinentes disponibles, las mujeres se encargan al menos dos veces y media más de estas labores que los hombres. (Organización Internacional del Trabajo [OIT], 2016). Para México, de acuerdo con el INEGI (2015) las mujeres mexicanas laboran $20,6 \%$ más horas de trabajo total que los hombres, $65 \%$ de su trabajo se integra por actividades no remuneradas del hogar y tan solo el $32,3 \%$ de su trabajo total se destina al trabajo remunerado.

Como se ha venido exponiendo, la falta de armonización del binomio familiatrabajo tiene un impacto negativo en la vida de las mujeres, un ejemplo de esto es el deterioro de su salud física y mental resultado de la sobrecarga de responsabilidades justificada bajo estereotipos de género que forjan expectativas sociales sobre su comportamiento respecto al cuidado familiar. En este sentido, se ha encontrado que los conflictos trabajo-familia están asociados a diferentes niveles de estrés (Biedma \& Medina, 2014; Rodríguez \& Aguilar, 2014) y estatus de salud física y mental (Martínez, 2003; Prieto \& Pérez, 2013). De tal manera que, cuando la distribución de las responsabilidades del hogar recae mayoritariamente en las mujeres estas presentan niveles significativos de ansiedad, depresión y baja autoestima (Blanco \& Feldman, 2000).

Sin embargo, lo que nos parece fundamental son los procesos subjetivos que dan paso a la culpabilización individual de la que habla Fernández-Villanueva (2010), refiriéndose a la paradoja en la que se encuentran las madres trabajadoras: ser profesionalmente competentes sin descuidar todo lo que demanda el ámbito privado. Esto lleva a las mujeres a experimentar culpa por no cumplir con las expectativas que implica ser "buena madre", pues dicha exigencia requiere, según los estereotipos, estar de tiempo completo en el hogar, así que, si las mujeres deciden trabajar fuera de casa sus posibilidades reales en cuanto a tiempo y apoyo son escasas, generando estrés constante al autoexigirse cumplir exitosamente en ambos escenarios, lo que conlleva a un deterioro corporal y emocional que da paso a sintomatologías relacionadas con el desgaste y el cansancio (Contreras Tinoco \& Castañeda Rentería, 2016).

No queremos dejar de lado el mencionar que el trabajo asalariado tiene aspectos positivos para la vida de las mujeres; es una condición que no solo aumenta sus conocimientos, habilidades, aptitudes o actitudes, dentro y fuera de su entorno de trabajo, sino que incrementa su sentido de legitimidad al sentirse reconocidas por su entorno (Cruz del Castillo, 2013). Da 
paso a la posibilidad de construir una feminidad más allá de los mandatos esposa-madre, sujetos con agencia personal responsables de sus propias vidas (Castañeda Rentería, 2019).

De ahí el interés del presente estudio, cuyo propósito es evaluar la Escala de Conciliación Vida-Familia (ECVF), a fin de establecer la estructura que lo determina y el criterio de confiabilidad (Alfa de Cronbach) a partir de muestras obtenidas. Con base en la información adquirida, se desea generar conocimiento basado en investigación que oriente el mejoramiento de la calidad de vida de las mujeres trabajadoras, tomando en cuenta que su ingreso a la esfera pública se da bajo el cobijo de estereotipos de género que dificultan el equilibrio de los escenarios familia-trabajo, repercutiendo negativamente en varias áreas de su vida.

\section{MÉTODO}

\subsection{Diseño}

Se trata de un estudio cuantitativo, con alcance explicativo sobre las propiedades psicométricas y estructura interna de una escala para medir la interferencia entre la vida laboral y familiar de mujeres con trabajo remunerado.

\subsection{Participantes}

Se incluyeron a 554 mujeres; el criterio de selección mediante un muestreo intencional, tomó en cuenta que las participantes actualmente desempeñaran una actividad laboral remunerada, formal o informal. Las mujeres se seleccionaron de dos ciudades, de dos estados diferentes de la república mexicana, 419 casos de la ciudad de Saltillo, Coahuila, y 135 casos de la ciudad de Hermosillo, Sonora.

Cabe señalar que una de las razones de centrar el estudio en estos dos contextos, es que ambas son ciudades fronterizas cuya característica es el rápido crecimiento económico proveniente de la inversión extranjera directo (IED) en los últimos 20 años. Coahuila y Sonora tuvieron tasas de crecimiento promedio en el periodo mucho más aceleradas que el total nacional (Mendoza, 2010). Esto ha permitido un aumento en la incorporación de las mujeres al mercado laboral. Para el caso de Coahuila, su participación económicamente activa (PEA) es de 37,1 \%, mientras que para Sonora es de 40,1\%, esta última por encima de la media nacional. En ambos estados, cerca del $34 \%$ de la PEA percibe dos salarios mínimos o menos, en el caso del género femenino esta proporción es del 41 \% (Secretaría del Trabajo y Previsión Social [STPS], 2016a, 
2016b). Es decir, cada vez vemos más mujeres participando en la economía del país, pero bajo condiciones laborales poco favorables.

Respecto a los datos socioeconómicos de las entrevistadas, en referencia a su lugar de trabajo 55,2 \% ( $n=306)$ se desempeñaba en el sector público, 37,9 \% $(n=210)$ en el sector privado y $6,8 \%(n=38)$ en organismos no gubernamentales. La edad promedio de las mujeres fue de 39 años, con una desviación típica de 11. La edad mínima fue de 18 y la máxima presente fue 66 años. En cuanto al estado civil y conyugal, $45,8 \%(n=254)$ de las mujeres dijo que es madre soltera, $27,8 \%(n=154)$ se encuentran casadas, $14 \%(n=82)$ son divorciadas, $8,5 \%(n=47)$ están solteras y el 1,8 \% $(n=10)$ dijo vivir en unión libre. El 36,5\% ( $n=202$ ) de la muestra tiene estudios universitarios, 20,4 \% ( $n=113$ ) tiene alguna carrera técnica, $16,6 \%$ ( $n=92)$ estudió hasta preparatoria o bachillerato, $12,8 \%$ ( $n=71)$ cuenta con secundaria, 4,2 \% concluyó solo la educación primaria $(n=23)$, solo el 9,4 \% $(n=52)$ reportó estudios de posgrado.

Entre los criterios de inclusión del estudio estaba ser mujeres mayores de edad, tener como mínimo un año de antigüedad en un trabajo formal asalariado de cualquier sector productivo, contar con pareja independiente al estado conyugal y que al menos tuvieran un hijo.

\subsection{Instrumentos}

Se utilizó un instrumento de 35 reactivos y un apartado de datos generales con 13 indicadores denominado Escala de Conciliación Vida-Trabajo (Hernández Montaño \& González Tovar, 2016). Este instrumento fue diseñado ad hoc para la investigación y con el propósito de validarse; se organizó en ocho dimensiones que abarcan: desarrollo personal, vida familiar, vida social, cuidado personal, alimentación, actividad física, satisfacción e interferencia. La forma de respuesta fue mediante una escala de frecuencias con cinco opciones para cada dimensión que van de Nunca (0) hasta Siempre (4). Además, fue sometido a revisión de dos expertos para validar el contenido, posteriormente se llevó a cabo el piloteo de la prueba con 30 casos, de la cual se obtuvo un coeficiente alfa de Cronbach de 0,828 y una correlación promedio inter-elementos de 0,125.

\subsection{Procedimiento}

La recolección de datos tuvo lugar en dos ciudades del norte del país, Hermosillo y Saltillo, México. El primer contacto se hizo con los departa- 
mentos de Recursos Humanos de las organizaciones participantes, se les envió vía electrónica una presentación ejecutiva de los objetivos y condiciones del estudio. Después de obtener las autorizaciones correspondientes se asistió a los centros de trabajo de las participantes dentro de sus horarios laborales, recabando la información de forma individual y en formato de autorreporte.

A cada entrevistada se le explicó el objetivo del estudio y que los datos serían utilizados para una investigación científica, que estos serían tratados con la máxima confidencialidad y anonimato, esto de acuerdo con las normas éticas de investigación de la Sociedad Mexicana de Psicología (2007).

Previo a la aplicación del instrumento, los encuestadores fueron capacitados con el propósito de uniformar el método de recogida de los datos, el cual incluyó la forma de administrar el instrumento.

\subsection{Análisis de datos}

Previa exploración de la distribución de los datos, estos se procesaron mediante análisis factorial exploratorio para determinar la estructura factorial. Se utilizó el método de principales componentes con una rotación ortogonal Varimax con Kaiser. Se exploró la adecuación de la matriz de correlaciones mediante la prueba de Káiser-Meyer-Olkin, que resultó con un valor de 0,864, la Prueba de Esfericidad de Bartlett rechazó la hipotesis nula (x2=5063,227, $p=0,000)$. Estos procedimientos fueron hechos en el paquete estadístico SPSS versión 21 y se confirmó la estructura factorial con el método de Mínimos Cuadrados Generalizados (GLS) con el paquete Statistica 10, se obtuvieron índices de bondad de ajuste: Función de Discrepancia (FD), el Error del Residuo Estandarizado (RMS), Índice de Bondad de Ajuste de Jöreskog (GFI), Índice de Bondad de Ajuste Corregido de Jöreskog (AGFI), Raíz Cuadrada de la Media de los Errores de Aproximación de Steiger-Lind (RMSEA).

\subsection{Conflicto de interés}

Los autores declaran que en desarrollo de este trabajo no se presentó ningún conflicto de intereses.

\section{RESULTADOS}

Se obtuvo una solución de seis componentes después de la rotación, con un total de varianza de 60,298 \%. Los componentes superaron el criterio de Káiser (Moral de la Rubia, 2006) para la extracción con autovalores iniciales 
iguales o mayores a uno. Inicialmente el instrumento se conformó en ocho apartados; sin embargo, después del análisis se consiguió una reducción de dimension a seis, con un porcentaje de explicación total de varianza de $60 \%$. Cada componente aporta entre el $6 \%$ y $14 \%$ de la explicación de la varianza después de la rotación. Después de realizar el procedimiento de rotación de los componentes se mantuvo una solución con seis factores. Se empleó una rotación con el método ortogonal Varimax para obtener las puntuaciones factoriales más altas en cada uno de los componentes y así explicarlas mejor. Mediante esta rotación se logró mayor claridad para la interpretación de los factores en comparación con un método oblicuo, que además no demostró correlaciones significativas entre los componentes. En la tabla 1 se presentan los autovalores y la varianza total explicada para la solución factorial obtenida.

\section{Tabla 1}

Resumen de autovalores y varianza explicada para una solución de seis componentes

\begin{tabular}{ccccccc}
\hline \multirow{2}{*}{ FACTOR } & \multicolumn{3}{c}{ AUTOVALORES INICIALES } & \multicolumn{3}{c}{$\begin{array}{r}\text { SUMA DE LAS SATURACIONES AL CUA- } \\
\text { DRADO DE LA ROTACIÓN }\end{array}$} \\
\cline { 2 - 7 } & TOTAL & $\begin{array}{c}\text { \% DE LA } \\
\text { VARIANZA }\end{array}$ & $\begin{array}{c}\% \\
\text { ACUMULADO }\end{array}$ & TOTAL & $\begin{array}{c}\text { \% DE LA } \\
\text { VARIANZA }\end{array}$ & $\begin{array}{c}\% \\
\text { ACUMULADO }\end{array}$ \\
\hline GRA & 6,134 & 24,536 & 24,536 & 3,504 & 14,017 & 14,017 \\
INT & 2,665 & 10,660 & 35,197 & 2,855 & 11,421 & 25,438 \\
PYC & 2,232 & 8,929 & 44,126 & 2,476 & 9,905 & 35,342 \\
CS & 1,732 & 6,927 & 51,053 & 2,461 & 9,843 & 45,186 \\
RH & 1,224 & 4,896 & 55,949 & 2,141 & 8,566 & 53,752 \\
TL & 1,087 & 4,350 & 60,298 & 1,637 & 6,547 & 60,298 \\
\hline
\end{tabular}

Nota: Elaboración propia. Método de extracción por componentes principales. GRA=gratificación, INT=interferencia, $P Y C=$ pareja y crianza, $C S=$ cuidado de la salud, $\mathrm{RH}=$ responsabilidades en el hogar, $\mathrm{TL}=$ tiempo libre .

En la tabla 2 se presentan las cargas factoriales por reactivo para los dos primeros componentes, además se reporta el nivel de confiabilidad a través del coeficiente de alfa de Cronbach y la correlación promedio inter-elementos. El primer componente se etiquetó como gratificación, el cual se integró con siete indicadores del instrumento y se refiere en su estructura a la satisfacción y percepción que las mujeres tienen de su trabajo, es decir, las ganancias personales y profesionales en su vida productiva. En particular se incluye la posibilidad de capacitarse y aprender cosas nuevas, la sensación de sentirse útiles y reconocidas. Además de lo anterior, ampliar su círculo de amistades y mantener una relación cercana con sus compañeros/as de trabajo, son elementos que forman parte de la evaluación de gratificación de las mujeres encuestadas. 
El segundo componente es interferencia; se agruparon cinco reactivos relacionados con el cruce entre las actividades laborales y las responsabilidades de ámbito privado. Estos evalúan la disposición de tiempo suficiente para el cuidado de la higiene e imagen personal y la preparación de alimentos.

El tercer componente se etiquetó como pareja y crianza, el cual supone la medición del nivel de conflicto que se genera en la relación de pareja al salir de casa a trabajar y asumir los compromisos "extras" que se derivan, como los viajes de trabajo o salidas tarde, además de la dificultad para equilibrar el tiempo que se dedica a los/as hijos/as y la vida productiva. El cuidado de la salud es el nombre del cuarto componente. Mide la posibilidad de llevar a cabo actividades físicas y mantener una dieta balanceada como parte de su autocuidado.

Tabla 2

Matriz de cargas factoriales de la escala de conciliación vida-trabajo

\begin{tabular}{|c|c|c|}
\hline \multirow{2}{*}{ REACTIVO } & \multicolumn{2}{|c|}{ COMPONENTE } \\
\hline & GRA & INT \\
\hline Ampliar mi círculo de amistades & 0,757 & \\
\hline Aprender cosas que me sirvan en mi vida diaria & 0,748 & \\
\hline Capacitarme constantemente & 0,664 & \\
\hline Tener una relación cercana con mis compañeras/os de trabajo & 0,663 & \\
\hline Sentirme útil y reconocida & 0,632 & \\
\hline Mantener una relación cercana con amigas/os & 0,596 & \\
\hline Contar con recurso económico para realizar actividades recreativas y/o culturales & 0,517 & \\
\hline Alfa de Cronbach & 0,839 & \\
\hline Correlación elemento-total & 0,427 & \\
\hline Tiempo suficiente para el cuidado de mi higiene personal & & 0,737 \\
\hline $\begin{array}{l}\text { Dedicar tiempo suficiente para la preparación de alimentos equilibrados en casa (plato } \\
\qquad \text { del buen comer) }\end{array}$ & & 0,724 \\
\hline Tiempo suficiente para el cuidado de mi imagen personal & & 0,714 \\
\hline Disponer de tiempo para planear las compras de alimentos semanalmente & & 0,647 \\
\hline Contar con recurso económico para comprarme algún artículo personal & & 0,581 \\
\hline Alfa de Cronbach & & 0,798 \\
\hline Correlación elemento-total & & 0,441 \\
\hline
\end{tabular}

Nota: Método de extracción por componentes principales. GRA=gratificación, INT= interferencia.

En la tabla 3 se presenta la matriz de cargas factoriales para tres componentes, así como el nivel de fiabilidad y la correlación inter-elementos. El componente cinco se integró por dos indicadores que representan el nivel de involucramiento de la pareja en las tareas domésticas y su conformidad con que la mujer trabaje fuera de casa, por lo que se decidió nombrar al compo- 
nente como responsabilidades en el hogar. El último factor llamado tiempo libre aporta una evaluación sobre la interrupción del goce del tiempo libre por actividades laborales y/o familiares.

En general, la carga factorial de mayor peso fue de 0,757 y se encuentra en el componente nombrado gratificación, mientras que la carga factorial de menor peso fue de 0,517 también del componente ya mencionado. Estas cargas factoriales se consideran adecuadas para el tamaño de la muestra, según Hair, Anderson, Tatham y Black (2007).

Tabla 3

Matriz de cargas factoriales de la Escala de conciliación vida-trabajo (Continuación)

\begin{tabular}{|c|c|c|c|c|}
\hline \multirow{2}{*}{ REACTIVO } & \multicolumn{4}{|c|}{ COMPONENTE } \\
\hline & PYC & CS & $\mathrm{RH}$ & TL \\
\hline $\begin{array}{l}\text { Existen conflictos con mi pareja por cuestiones laborales (salidas tarde, viajes, exceso de } \\
\text { trabajo) }\end{array}$ & 0,696 & & & \\
\hline $\begin{array}{l}\text { Mi pareja considera que sería mejor que me dedicara a cuidar a los hijos/as y atender la } \\
\qquad \text { casa en vez de trabajar }\end{array}$ & 0,694 & & & \\
\hline Las horas que dedico a mis hijos/as y familia interfieren con mi trabajo & 0,648 & & & \\
\hline He tenido que rechazar una promoción o traslado laboral por razones familiares & 0,630 & & & \\
\hline Las horas de trabajo interfieren con el tiempo que debo dedicar a mis hijos/as y familia & 0,590 & & & \\
\hline Alfa de Cronbach & 0,702 & & & \\
\hline Correlación elemento-total & 0,314 & & & \\
\hline $\begin{array}{l}\text { Tener tiempo para realizar alguna actividad física como deporte, zumba, aerobics, baile, } \\
\text { etc. }\end{array}$ & & 0,847 & & \\
\hline Mantener mi condición física & & 0,796 & & \\
\hline Mantener una dieta balanceada & & 0,704 & & \\
\hline Alfa de Cronbach & & 0,791 & & \\
\hline Correlación elemento-total & & 0,555 & & \\
\hline Mi pareja se involucra en el cuidado de los niños/as de la forma que quisiera & & & 0,834 & \\
\hline Los quehaceres de la casa están distribuidos con mi pareja de la forma que quisiera & & & 0,771 & \\
\hline Mi pareja está de acuerdo con que trabaje & & & 0,621 & \\
\hline Alfa de Cronbach & & & 0,661 & \\
\hline Correlación elemento-total & & & 0,394 & \\
\hline El tiempo que dedico al trabajo interfiere con mi tiempo libre & & & & 0,821 \\
\hline
\end{tabular}


El tiempo que dedico a la familia interfiere con mi tiempo libre $\quad 0,800$

$\begin{array}{ll}\text { Alfa de Cronbach } & 0,733\end{array}$

Nota: Método de extracción por componentes principales. PYC= pareja y crianza, $\mathrm{CS}=$ cuidado de la salud, $\mathrm{RH}=$ responsabilidades en el hogar, $\mathrm{TL}=$ tiempo libre

La tabla 4 presenta los índices de bondad y ajuste para el análisis confirmatorio. En el AFC, se probó el modelo hipotético del instrumento con el método de Mínimos Cuadrados Generalizados, con las variables latentes correlacionadas y sin correlacionar. El modelo obtuvo un ajuste moderado en las dos versiones probadas. Los indicadores básicos como el coeficiente Chi cuadrado rechazaron la hipótesis nula, sin embargo, el valor de RMS fue cercano a cero. Los índices de bondad de ajuste fueron superiores a 0,80, la RMSEA fue inferior a 0,070, criterio mínimo aceptable para evaluar la bondad del modelo.

Tabla 4

Indicadores de bondad de ajuste para la estructura de la escala de conciliación vidafamilia para mujeres insertas en el mercado laboral

\begin{tabular}{cccc}
\hline & & \multicolumn{2}{c}{ ESTRUCTURA } \\
\cline { 2 - 3 } & INDICADORES & $\begin{array}{c}\text { CORRELA- } \\
\text { CIONADA }\end{array}$ & $\begin{array}{c}\text { SIN CORRE- } \\
\text { LACIONAR }\end{array}$ \\
\hline \multirow{3}{*}{ Básicos } & Función de Discrepancia (DF) & 1,662 & 1,789 \\
& Grados de libertad (gl) & 260 & 253 \\
No & Nivel de probabilidad (p) & 0,000 & 0,000 \\
centralidad & Error del Residuo Estandarizado (RMS) & 0,169 & 0,282 \\
& Índice de Bondad de Ajuste de Jöreskog (GFI) & 0,867 & 0,851 \\
& Índice Corregido de Bondad de Ajuste Jöreskog (AGFI) & 0,834 & 0,823 \\
\hline
\end{tabular}

Nota: GLS= Método por Mínimos Cuadrados Generalizados

\section{DISCUSIÓN}

Los resultados muestran que las variables de la ECVF para mujeres insertas en el mercado laboral del noreste de México relacionadas entre sí permiten reconocer aquellas que comparten significados comunes capaces de definir con mayor veracidad las respuestas dadas a las preguntas del instrumento en sus diferentes factores. Como se muestra, el método de validación empírica elaborado de manera exploratoria fue útil para el análisis de la sensibilidad estadística de la escala aplicado a la población estudiada.

La estructura de la ECVT obtuvo validez estructural en el análisis exploratorio, ya que fue posible realizar una reducción en el número de reactivos. La 
estructura factorial presentó un buen porcentaje de explicación de varianzas y las cargas factoriales aportaron un peso factorial medio. Originalmente la estructura del instrumento estaba constituida por ocho componentes, después del procesamiento se redujo a seis dimensiones que en su interpretación fueron congruentes. Además, hay que destacar que también se redujo el número de reactivos que integraron el instrumento, pasó de 35 a 25 . La consistencia interna mejoró con la reducción, pues paso de un coeficiente alfa de Cronbach de 0,843 a 0,874 . Esto es, hay una consistencia interna satisfactoria (Oviedo \& Campo-Arias, 2005).

Asimismo, la organización de dicha escala se puede relacionar con las categorías del Cuestionario Trabajo-Familia propuesto por Feldman (2001) (citado en Feldman et al., 2008), quien propuso los componentes: a) gratificación, entendido como la relación entre la satisfacción del mismo trabajo y los beneficios en el área de pareja y familia; b) interferencia, que implica la compaginación de tareas laborales con las tareas del hogar y el tiempo libre. Cabe destacar que esta propuesta es para el contexto de Caracas, Venezuela.

El instrumento utilizado en este estudio contiene temas cruciales sobre la conciliación vida-familia, siendo una escala breve que posibilita el ahorro de tiempo en la tarea de evaluar este constructo. Para las participantes, el componente de gratificación pone de manifiesto el efecto positivo de contar con una actividad asalariada, pues esta les ofrece la posibilidad de acceder a experiencias que las visibilice socialmente, proporcionando una sensación de utilidad y reconocimiento (Cruz del Castillo, 2013), lo que sugiere un beneficio personal y profesional al permitirles la posibilidad de acceder a conocimiento y habilidades para afrontar los retos profesionales.

Así mismo, salir de casa les da la oportunidad de mantener relaciones cercanas con amistades y compañeras de trabajo, quienes juegan un papel importante al convertirse en una red de apoyo con quien compartir las liviandades de la vida y temas más profundos (Fawaz-Yiss \& Rodríguez-Garcés, 2013), lo cual se incrementa cuando el salario les permite gozar de actividades recreativas durante su tiempo libre.

Para las trabajadoras, el componente crianza y pareja pone de manifiesto los conflictos que se suscitan en la relación por cuestiones laborales, principalmente cuando dedican más tiempo de lo usual a éste, pues esto implica restarle tiempo al cuidado de la familia y las labores domésticas, es decir, las mujeres que deciden optar por un trabajo asalariado lo hacen cargando 
a cuestas los estereotipos de género que les impone la compaginación de su presencia fuera y dentro del hogar muchas veces a costa del deterioro de su propia salud (Blanco \& Feldman, 2000; Contreras Tinoco \& Castañeda Rentería, 2016).

De ahí que el tema de la conciliación vida-trabajo cobra importancia frente al incremento de las mujeres en los sectores productivos (OIT, 2016), crea la necesidad de validar herramientas que evalúen la reconciliación entre las tareas laborales, domésticas, de crianza y de cuidado personal, así como sus consecuencias en la vida, mismos que permitirán la documentación de los avances en la edificación de una cultura organizacional de género.

Finalmente, no podemos hablar de equidad e igualdad de género solo por el hecho de que las mujeres figuren en la escena pública sin conocer en qué condiciones lo hacen. Existe la necesidad de promover transformaciones estructurales, sociales y culturales para que las estrategias de conciliación no sean solo de las mujeres. Construir la equidad en un contexto privado no se puede lograr sin tomar en cuenta cómo se encuentra la equidad en el nivel social, cultural e institucional, pues no radica en un trabajo meramente individual, sino que nos requiere a todas y todos en los diferentes niveles.

Financiamiento: Estrategias de conciliación vida-trabajo en mujeres con trabajo remunerado, Universidad Autónoma de Coahuila.

Agradecimientos: A la Universidad Autónoma de Coahuila.

\section{REFERENCIAS}

Alarcón, I.L. (2012). Conciliación de la vida familiar y laboral en parejas heterosexuales. La ventana, 4(35), 58-92. Recuperado de: http://www.scielo.org.mx/ scielo.php?script=sci_arttext\&pid=S140594362012000100004\&Ing=es\&tlng=es

Alcaniz, M. (2015). Género con clase: la conciliación desigual de la vida laboral y familiar. Revista Española de Sociología, (23), 29-55. Disponible en http://www. fes-sociologia.com/files/res/23/02.pdf

Bamberry, L. (2016). Restructuring women's work; labour market and household gender regimes in the Greater Latrobe Valley, Australia, Gender, Place \& Culture, 23(8), 1135-1149. http://dx.doi.org/10.1080/0966369X.2015.1090408

Benería, L. (2006). Trabajo productivo/reproductivo, pobreza y políticas de conciliación. Nómadas (Col), (24), 8-21. Disponible en http://www.cieg.unam.mx/ lecturas_formacion/relaciones_genero/modulo_5/M5_S2_L1.pdf

Biedma, J.M. \& Medina, J.A. (2014). Impacto de la conciliación laboral y familiar en el desempeño organizativo. Intangible Capital, 10(3), 448-466. http://dx.doi. org/10.3926/lc.506 
Blanco, G. \& Feldman, L. (2000). Responsabilidades en el hogar y salud de la mujer trabajadora. Salud Pública de México, 42(3), 217-225. Disponible en http://www. redalyc.org/html/106/10642310/index.html

Campos, J., Ronda, E., Artazcoz, L. \& Benavides, F. (2012). Desigualdades de género en salud laboral en España. Gaceta sanitaria, 26(4), 343-351. http://dx.doi. org/10.1016/j.gaceta.2011.09.025

Castañeda Rentería, L. (2019). ¿Nuevas sujetas, nuevas identidades? La vivencia profesional en la configuración de la identidad de género. Noésis, (55), 88-108. https://doi.org/10.20983/noesis.2019.1.5

Contreras Tinoco, K., \& Castañeda Rentería, L. (2016). Tensiones entre el cuerpo productivo de la mujer y la normatividad de género en torno a la maternidad. Revista Latinoamericana de Estudios sobre Cuerpos, Emociones y Sociedad, 8 (21), 10-24. Disponible en http://www.redalyc.org/pdf/2732/273246916002.pdf

Cruz del Castillo, C. (2013). Mujeres trabajando: ¿por amor o independencia? En T. Rocha S., C. Cruz Del Castillo (Coords.), Mujeres en transición: Reflexiones teórico-empíricas en torno a la sexualidad, la pareja y el género (pp. 221-241). México: Universidad Iberoamericana.

Díaz, C., Dema, S. \& Finkel, L. (2015). Desigualdades de género en la distribución de los recursos económicos en las parejas. Papers, 100(1), 53-73. http://dx.doi. org/10.5565/rev/papers.591

Fawaz-Yiss, M.J. \& Rodríguez-Garcés, C. (2013). Mujeres rurales y trabajo en Chile Central. Actitudes, factores y significaciones. Cuadernos de Desarrollo Rural, 10(72), 47-68. Disponible en http://revistas.javeriana.edu.co/index.php/desarroIloRural/article/view/7014

Feldman, L., Vivas, E., Lugli, Z., Zaragoza, J. \& Gómez, V. (2008). Relaciones trabajo-familia y salud en mujeres trabajadoras. Salud Pública México, 50(6), 482-489. Disponible en http://www.redalyc.org/html/106/10611162007/

Fernández-Villanueva, C. (2010). La equidad de género: presente y horizonte próximo. Quaderns de Psicología, 12(2), 93-104. Disponible en https://www.quadernsdepsicologia.cat/article/view/809/714

Guevara, E. (2010). Cuando el amor se instala en la modernidad. Intimidad, Masculinidad y Jóvenes en México. UNAM/CEIICH.

Hair, J., Anderson, R., Tatham, R. \& Black, W. (2007). Análisis Multivariante. Madrid: Prentice Hall.

Hernández, A. (2016). Amor en tiempos de equidad. El caso de las y los jóvenes de Coahuila. México: Fontamara.

Hernández, A. \& González, J. (2016). Escala de Conciliación Vida-Trabajo, Facultad de Psicología, Universidad Autónoma de Coahuila, Saltillo, Coahuila, México.

Hernández, A., González, J., López, D. I. \& Saucedo, A.D. (2015). Relaciones de género en familias de Coahuila. Memorias del Congreso Mexicano de Psicología. (pp. 808-810). Cancún, Quintana Roo.

Hernández, A., González, J., Regino, D., Abril, E., Cubillas, M.J., Córdova, A. \& Domínguez, S. (2017). Diferencias de la conciliación vida-carrera en mujeres de Sonora y Coahuila, México. Diversitas: perspectivas en psicología, 13(1), 69-80. http://dx.doi.org/10.15332/s1794-9998.2017.0001.06

Instituto Nacional de Estadística y Geografía [INEGI] (2011). Encuesta Nacional sobre la Dinámica de las Relaciones en los Hogares (ENDIREH). México: INEGI.

INEGI (2015). Estadísticas a propósito del día internacional de la eliminación de la violencia contra la mujer. Datos nacionales. Recuperado de http://www.inegi.org. $\mathrm{mx} /$ saladeprensa/aproposito/2015/violencia0.pdf 
INEGI (2016). Estadística a propósito de la mujer (8 de marzo). Datos económicos nacionales. Recuperado de: http://www.inegi.org.mx/saladeprensa/ aproposito/2016/mujer2016_0.pdf

Instituto Nacional de las Mujeres (INEGI-INMUJERES) (2007). Mujeres y hombres en México. Segundo Trimestre 2006.

Izquierdo, M. J. (2013). La socialización de género. En C. Díaz y S. Dema (Coords.), Sociología y Género (pp.132-164). Madrid: Tecnos

Jiménez, A. \& Gómez, V. (2015). Conciliando trabajo-familia: análisis desde la perspectiva de género. Diversitas: perspectivas en psicología, 11(2), 289-302. http://dx.doi.org/10.15332/s1794-9998.2015.0002.09

López, E., Ponce, M., Findling, L., Lehner, P., Venturiello, M.P., Mario, S. \& Champalbert, L. (2011). Mujeres en tensión: la difícil tarea de conciliar familia y trabajo. Población de Buenos Aires, 8(13), 7-25. Disponible en http://www.redalyc.org/ pdf/740/74018029001.pdf

Martínez, I. (2003). Los efectos de las asimetrías de género en la salud de las mujeres. Anuario de Psicología, 34(2), 253-266. Disponible en http://revistes.ub.edu/ index.php/Anuario-psicologia/article/viewFile/8750/10942

Mendoza, J.E. (2010). El mercado laboral en la frontera norte de México: estructura y políticas de empleo. Estudios fronterizos, 11(21), 9-42. Recuperado de http://www. scielo.org.mx/scielo.php?script=sci_arttext\&pid=S0187-69612010000100001\&Ing=es\&tlng=es

McMillan, H. S., Morris, M. L., \& Atchley, K. E. (2011). Constructs of the work/life interface. A synthesis of the literature and introduction of the concept of work/ life harmony. Human Resource Development Review, 10(1), 6-25. http://doi. org $/ 10.1177 / 1534484310384958$

Moral de la Rubia, J. (2006). Análisis factorial exploratorio y su aplicación al desarrollo de escalas. En M.T. González Ramírez y R. Landeros Hernández (Eds.), Estadística con SPSS y metodología de la investigación (pp. 387-437). México: Trillas.

Organización para la Cooperación y el Desarrollo Económico [OCDE] (2015). Balance vida-trabajo. Recuperado de http://www.oecdbetterlifeindex.org/es/topics/ work-life-balance-es/

Organización Internacional del Trabajo [OIT] (2016). Tendencias Mundiales del Empleo de las Mujeres. Recuperado de http://www.ilo.org/public/english/employment/ strat/download/getb07sp.pdf

Oviedo Heid, C. \& Campo Arias, A. (2005). Aproximación al uso del coeficiente de Alfa de Cronbach. Revista Colombiana de Psiquiatría, 34(4), 572-580. Disponible en http://www.redalyc.org/comocitar.oa?id=80634409

Pérez, M., Vela, M., Abella, S. \& Martínez, A. (2015). El enriquecimiento trabajo-familia: nuevo enfoque en el estudio de la conciliación y la satisfacción laboral de los empleados. Universia Business Review, (45), 16-33. Disponible en https://ubr. universia.net/article/viewFile/1169/1450

Prieto, C. \& Pérez, S. (2013). Desigualdades laborales de género, disponibilidad temporal y normatividad social. Revista Española de Investigaciones Sociológicas (REIS), 141(1), 113-132. http://dx.doi.org/10.5477/cis/reis.141.113

Rocha, T. E. (2013). Mujeres, pareja y familia: la transformación de roles y la búsqueda de la equidad. En T. Rocha S. y C. Cruz Del Castillo (Coords.), Mujeres en transición: Reflexiones teórico-empíricas en torno a la sexualidad, la pareja y el género (pp.181-217). México: Universidad Iberoamericana. 
Rodríguez, P. \& Aguilar, H. (2014). Estrés laboral en mujeres trabajadoras y amas de casa. Jóvenes en la Ciencia, 1(1), 459-463. Recuperado de: http://www.jovenesenlaciencia.ugto.mx/index.php/jovenesenlaciencia/article/view/687/pdf2

Sagastizabal, M. \& Legarreta, M. (2016). La triple presencia-ausencia: una propuesta para el estudio de trabajo doméstico-familiar, el trabajo remunerado y la participación sociopolítica. Papeles del CEIC, 1(151). http://dx.doi.org/10.1387/ pceic.15447

Secretaría del Trabajo y Previsión Social [STPS]. (2016a). Información laboral para Sonora. Subsecretaría de empleo y productividad laboral. Disponible en http:// www.estadisticasonora.gob.mx/archivos/file/46_REVISTA_1erTRIM_2016_ INTER.pdf

STPS. (2016b). Información laboral para Coahuila. Subsecretaría de empleo y productividad laboral. Disponible en http://www.estadisticasonora.gob.mx/archivos/ file/46_REVISTA_1erTRIM_2016_INTER.pdf

Sociedad Mexicana de Psicología (2007). Código ético del psicólogo. México: Trillas.

Tena, O. (2013). Estrategias utilizadas por mujeres policías para conciliar sus deberes de trabajo doméstico y remunerado: ¿Un camino hacia la equidad? Acta Colombiana de psicología, 16(2) 81-91. http://dx.doi.org/10.14718/ACP.2013.16.2

Tobío, C. (2012). Cuidado e identidad de género. De las madres que trabajan a los hombres que cuidan. Revista Internacional de Sociología (RIS), 70(2), 399-422. http://dx.doi.org/10.3989/ris.2010.08.26

Worth, N. (2016). Who we are at work: millennial women, everyday inequalities and insecure work. Gender, Place \& Culture, 23(9), 1302-1314. http://dx.doi.org/10.1 080/0966369X.2016.1160037

\section{(cc) BY}

